\title{
A model-based approach to the computation of area probabilities for precipitation exceeding a certain threshold
}

\author{
B. Kriesche $^{\text {a }}$, A. Koubek ${ }^{\mathrm{b}}$, Z. Pawlas ${ }^{\mathrm{b}}$, V. Beneš ${ }^{\mathrm{b}}$, R. Hess ${ }^{\mathrm{c}}$ and V. Schmidt $^{\mathrm{a}}$ \\ a Ulm University, Institute of Stochastics, Ulm, Germany
${ }^{\mathrm{b}}$ Charles University in Prague, Faculty of Mathematics and Physics, Department of Probability and \\ Mathematical Statistics, Prague, Czech Republic \\ ${ }^{\mathrm{c}}$ Deutscher Wetterdienst, Research and Development, Offenbach, Germany \\ Email: bjoern.kriesche@uni-ulm.de
}

\begin{abstract}
In order to provide consistent weather warnings it is essential to estimate the probabilities for certain weather events occurring. There are a number of operational numerical and statistical methods for estimating the probability that precipitation occurs at a fixed location (a point probability). However, there is a growing interest in the computation of probabilities for precipitation occurring somewhere in a geographical region (an area probability). An example would be the area of responsibility of a fire department, where an emergency occurs when there is intense rain anywhere within that area. The derivation of such area probabilities is difficult and no applicable method for their computation is known. In literature, some theoretical relationships between point and area probabilities are discussed under very simplifying assumptions. Furthermore, several stochastic models for precipitation cells have been proposed with none of them being suitable for our purposes due to restrictions such as e.g. spatial and temporal stationarity or inappropriate (or missing) model fitting. Recently, we proposed a (less restrictive and more robust) model-based approach to the computation of area probabilities for the occurrence of precipitation. This method is based on a non-stationary stochastic model for precipitation cells (basically a Cox germ-grain model with circular grains), which is characterized by a sequence of local intensities for the formation of precipitation cells and a cell radius. Furthermore, we proposed an approach for the algorithmic computation of all model characteristics from a sequence of point probabilities and described how area probabilities can be computed by repeated simulation of that model. In the present paper, we discuss an extension of this model by additionally including precipitation amounts. The available data contains sequences of point probabilities for precipitation exceeding various thresholds. At first, a gamma distribution is fitted to obtain moments of point precipitation amounts. Then, we briefly recall our model for the representation of precipitation cells. The most important and most recent results address the spatial stochastic modeling of precipitation amounts, which is done by assigning a response function to each precipitation cell. The response functions are then multiplied by random scaling factors and summed up to obtain precipitation amounts. We derive formulas for the expectation and variance of random point precipitation amounts in our model. Comparing the computed moments with those obtained from the data allows us to fit the distributions of the random scaling variables. Finally, area probabilities for the occurrence of precipitation exceeding a given threshold are estimated by repeated simulation of the precipitation model. We evaluate our results by analyzing how the choice of the response function and the distribution of scaling variables influence estimated point and area probabilities. The novelty of the presented approach is that, for the first time, a widely applicable estimation of area probabilities is possible, which is based solely on predicted point probabilities (i.e., no precipitation observations are needed). Furthermore, the method works in a quite reasonable computation time, which makes it suitable for applications in modern weather prediction.
\end{abstract}

Keywords: Probabilistic weather prediction, area probabilities, precipitation amounts, spatial stochastic model, Monte Carlo simulation 
B. Kriesche et al., A model-based approach to the computation of area probabilities ...

\section{INTRODUCTION}

An important responsibility of weather services such as Deutscher Wetterdienst (DWD) is to provide accurate and reliable weather warnings. Of particular interest is the computation of probabilities for the occurrence of dangerous weather events, e.g., strong wind gusts or heavy precipitation. Existing methods as used by DWD are designed to compute point probabilities, i.e., probabilities for the occurrence of a weather event at a given point. In order to improve the warning system it is desirable to establish warnings that arise if the harmful weather event occurs anywhere in a defined area (instead of at a fixed point). The probabilities such warnings are based on are called area probabilities and they are always larger than a point probability at any fixed point within the area. General relationships between point and area probabilities are still unclear. We expect that point probabilities not only provide information about the weather situation at the corresponding locations but also about surroundings due to their spatial correlations. The exact relationships, however, are not known. In Krzysztofowicz (1998) theoretical relationships between point and area probabilities have been established under simplifying assumptions (circular precipitation cells and forecast areas, uniformly distributed precipitation cells), which make these approaches inappropriate for most practical purposes.

In the present paper, we describe a model-based method to compute area probabilities for the occurrence of precipitation exceeding a given threshold. The model can be divided into two parts: (1) modeling the occurrence of precipitation cells and (2) the modeling of precipitation amounts. A few approaches to spatially continuous modeling of precipitation cells have been described in the literature, see e.g. Onof et al. (2000), Wheater et al. (2005) or Segond and Onof (2009). They all provide valuable ideas but are subject to certain limitations (spatial stationarity of rain events, model fitting only possible to observed radar data, no consideration of area probabilities), which prevent the application of such models for the automated generation of weather warnings on a nation-wide scale. In Kriesche et al. (2015), we proposed a more robust and less restrictive model for precipitation cells, where all model characteristics can be determined algorithmically from a sequence of point probabilities. Repeated simulation of the model allows for the computation of area probabilities for the occurrence of precipitation in arbitrary areas of interest. Unfortunately, this method is not directly applicable to compute area probabilities for the occurrence of precipitation amounts exceeding a certain threshold, which is of major interest when generating weather warnings. Thus, the most natural next step is to develop a model for precipitation amounts. In Rodriguez-Iturbe et al. (1986), a complex model for both precipitation cells and precipitation amounts is proposed, where circular precipitation cells occur according to a stationary Poisson or cluster process and precipitation amounts are modeled by various response functions. Moreover, Rodriguez-Iturbe et al. (1986) focus on the derivation of several theoretical properties and the comparison of different model specifications. Unfortunately, no fitting of the model to observed data is described and no attempt to use this model for the generation of weather forecasts is made. Although their paper contains some valuable ideas for the modeling of precipitation amounts, the presented approaches are not yet suitable to be used for the automated generation of weather warnings. One possible step towards this goal is presented in the following.

\section{POINT PRECIPITATION PROBABILITIES AND AMOUNTS}

The statistical method for the computation of area probabilities presented in this paper is based on point probabilities computed for a set of geographically distinct locations. At DWD, point probabilities are determined in a two-step procedure. At first, a deterministic weather forecast is made based on the numerical weather prediction model GME (Majewski et al., 2002) and the Integrated Forecasting System of the European Centre for Medium-Range Weather Forecasting (IFS/ECMWF). In a second step systematic errors of these models are statistically reduced and point probabilities are derived with model output statistics (Wilks, 1995) using historical meteorological observations from about 1600 weather stations worldwide.

In this study we consider a set of 503 synoptic weather stations located in Germany and Luxembourg. To address seasonal changes in precipitation events a summer period (June/July 2012) and a winter period (November/December 2012) are selected. At each day of this time period point probabilities are computed for seven one-hour forecast steps (02-03 UTC, 05-06 UTC, .., 20-21 UTC) resulting in a total of 854 forecast steps. For each weather station and each forecast step, point probabilities for the occurrence of precipitation of more than $u \mathrm{~mm}$ are derived using model output statistics for a sequence of thresholds $u=0,0.1,0.2,0.3,0.5,0.7,1,2,3,5,10,15$. For $u=0$ the corresponding probability represents the chance of precipitation of any amount.

It may happen that in our data point probabilities for a fixed weather station and forecast step are not mono- 

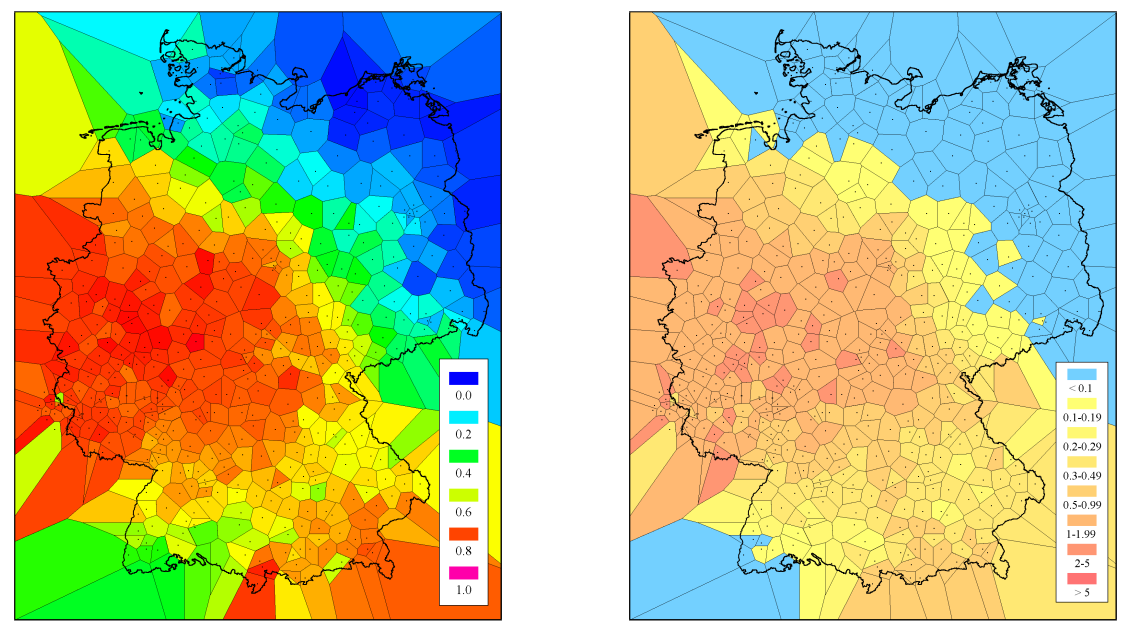

Figure 1. Sample data for June 13, 2012, 05-06 UTC: the locations of the 503 weather stations and the corresponding Voronoi tessellation (see Sect. 3.1), where each Voronoi cell is colored according to the point probability for the occurrence of precipitation (left) and the expected point precipitation amount (right).

tonically decreasing with increasing threshold $u$, e.g., the probability for precipitation of more than $0.7 \mathrm{~mm}$ may be higher than the probability for precipitation of more than $0.5 \mathrm{~mm}$. This can occur since available point probabilities are estimated for each threshold $u$ separately and the computation is subject to statistically independent noise, which sometimes results in the described inconsistencies. Furthermore, information about the moments of precipitation amounts would be more appropriate for model fitting as we show in Sect. 3. In order to overcome both problems, we fit a gamma distribution, as suggested by Wilks (1995), to point precipitation amounts by minimizing the absolute differences between the tail function of the gamma distribution and the available (possibly non-monotonic) point probabilities. The result is a sequence of consistent (monotonically decreasing) point probabilities derived from the fitted gamma distribution for the thresholds given above together with the expectation and variance of precipitation amounts for each weather station and each forecast step. Fig. 1 illustrates some sample data of this kind.

\section{AREA PRECIPITATION PROBABILITIES}

\subsection{Model for the occurrence of precipitation}

We briefly recall a model for the representation of precipitation cells, which was introduced in Kriesche et al. (2015) for the computation of area probabilities regarding the occurrence of precipitation. This model forms a basis for the modeling of precipitation amounts in Sect. 3.2. We always consider a fixed forecast step in the following. Our data includes a sequence $p_{s_{1}}, \ldots, p_{s_{503}}$ of point probabilities for the occurrence of precipitation at the locations $s_{1}, \ldots, s_{503} \in W$ of the 503 weather stations. The rectangular observation window $W \subset \mathbb{R}^{2}$ contains the boundaries of Germany and Luxembourg. We interpret our data as realizations of a random field $\left\{P_{t}, t \in W\right\}$ at the locations $s_{1}, \ldots, s_{503}$, i.e., a family of random variables, where $P_{t}$ denotes the random point probability at location $t \in W$. As the fundamental assumption of our modeling approach we consider the following intuitive relationship between precipitation probabilities and the occurrence of precipitation. We suppose that there is precipitation at location $t \in W$ if and only if $t$ is covered by a random precipitation set $M \subset W$, which we assume to be the union of all precipitation cells located within our observation window. Unfortunately, precipitation sets can have very complicated structures, which makes some simplifications necessary. We follow previous approaches from the literature (Rodriguez-Iturbe et al., 1986; Wheater et al., 2005) by assuming that precipitation cells occur according to a Poisson-based point process and have a random cell size. For that purpose, we introduce a piecewise constant random intensity function $\left\{\Lambda_{t}, t \in W\right\}$, which is given by

$$
\Lambda_{t}=\sum_{i=1}^{503} A_{i} I_{V\left(s_{i}\right)}(t) \quad \text { for all } t \in W
$$


B. Kriesche et al., A model-based approach to the computation of area probabilities ...

Here, $I_{V}: W \rightarrow\{0,1\}$ denotes the indicator of the set $V \subset W$ and $\left\{V\left(s_{1}\right), \ldots, V\left(s_{503}\right)\right\}$ is the Voronoi tessellation induced by the point pattern $s_{1}, \ldots, s_{503}$ in $W$, where the Voronoi cell $V\left(s_{i}\right)$ is defined by

$$
V\left(s_{i}\right)=\left\{x \in W:\left|x-s_{i}\right|<\left|x-s_{j}\right| \text { for all } j=1, \ldots, 503 \text { with } j \neq i\right\} \quad \text { for } i=1, \ldots, 503 .
$$

The random variables $A_{1}, \ldots, A_{503}$ can be interpreted as some non-negative (local) random intensities, which means that precipitation cells are distributed according to the values of $A_{1}, \ldots, A_{503}$. In particular, $A_{i}$ is the (random) expected number of cells in a unit area located within the neighborhood of $s_{i}$. In the next step, the centers of precipitation cells are modeled by a spatial Cox point process $\left\{X_{i}, i=1, \ldots, Z\right\}$ in $W$ with random intensity function $\left\{\Lambda_{t}, t \in W\right\}$, see Chiu et al. (2013) for a definition, with $Z$ being the random total number of cells in $W$. Finally, we assume precipitation cells to be circular with some positive random radius $R$. Combining these assumptions the precipitation set $M$ can be represented as the union set of precipitation cells, i.e.,

$$
M=\bigcup_{i=1}^{Z} b\left(X_{i}, R\right)
$$

where $b(x, r)$ denotes the two-dimensional ball with center $x \in \mathbb{R}^{2}$ and radius $r>0$. Note that for a particular realization of $A_{1}, \ldots, A_{503}$ and $R$ the union set $M$ is called a Boolean model based on a Poisson process with deterministic intensity function and radius, see Chiu et al. (2013). Now, point and area probabilities for the occurrence of precipitation are modeled as hitting probabilities (e.g. $P\left(B \cap M \neq \emptyset \mid A_{1}, \ldots, A_{503}, R\right)$ for a test area $B \subset W$ ) of the union set $M$ conditioned on the local random intensities $A_{1}, \ldots, A_{503}$ and the random cell radius $R$. Closed formulas for conditional point and area probabilities can be derived by using the properties of the underlying Poisson process. Finally, the model characteristics $A_{1}, \ldots, A_{503}$ and $R$ have to be determined to allow for an effective computation of area probabilities. However, this is less important for the modeling of precipitation amounts (which is the main issue of the present paper) and can be looked up in Kriesche et al. (2015).

\subsection{Model for precipitation amounts}

The model for precipitation cells presented above is now extended by adding precipitation amounts. To simplify the notation we always consider fixed realizations $a_{1}, \ldots, a_{503}$ and $r$ of $A_{1}, \ldots, A_{503}$ and $R$ in the following. At first, we assign a response function with circular support to each precipitation cell $b\left(X_{i}, r\right)$, which models the precipitation amounts produced by this cell. Then, these functions are multiplied with some positive random scaling variables, where all precipitation cells with centers in the same Voronoi cell $V\left(s_{i}\right)$ are scaled by the same factor. This ensures that precipitation amounts generated by single precipitation cells can vary for different regions of Germany. Precipitation amounts at each location are obtained by summing the values of the response functions of all precipitation cells covering that location (if there is more than one). If there is no cell covering the considered location, then the precipitation amount is equal to 0 . This modeling approach is illustrated in Fig. 2. We now describe this model for precipitation amounts more mathematically, which is needed to allow for model fitting. Let $K: \mathbb{R}^{2} \rightarrow[0, \infty)$ be a symmetric response function with circular support defined as

$$
K(t)=\left(1-t^{\top} t\right)^{p} I_{\left\{t^{\top} t<1\right\}} \text { for } t \in \mathbb{R}^{2},
$$

where $p>0$ is a shape parameter. For $p=1$ the function $K(\cdot)$ is a scaled Epanechnikov kernel, for $p=2$ a scaled biweight kernel, for $p=3$ a scaled triweight kernel, and for $p=0.5$ the upper half of a unit ball. Furthermore, given $A_{1}=a_{1}, \ldots, A_{503}=a_{503}$ und $R=r$, we consider a sequence $C_{1}, \ldots, C_{503}$ of independent non-negative random scaling variables (for the 503 Voronoi cells $V\left(s_{1}\right), \ldots, V\left(s_{503}\right)$ ) that are additionally assumed to be independent of the point process $\left\{X_{i}, i=1, \ldots, Z\right\}$ of precipitation cell centers. According to the modeling approach described above we introduce the random field $\left\{\Gamma_{t}, t \in W\right\}$ of precipitation amounts, where

$$
\Gamma_{t}=\sum_{i=1}^{Z} \sum_{j=1}^{503} C_{j} I_{V\left(s_{j}\right)}\left(X_{i}\right) K\left(\frac{X_{i}-t}{r}\right) \quad \text { for } t \in W .
$$

Notice that the random field $\left\{\Gamma_{t}, t \in W\right\}$ is completely described by the precipitation cell parameters $a_{1}, \ldots, a_{503}$ and $r$, the random scaling variables $C_{1}, \ldots, C_{503}$ and the shape parameter $p$. As explained in 
B. Kriesche et al., A model-based approach to the computation of area probabilities ...

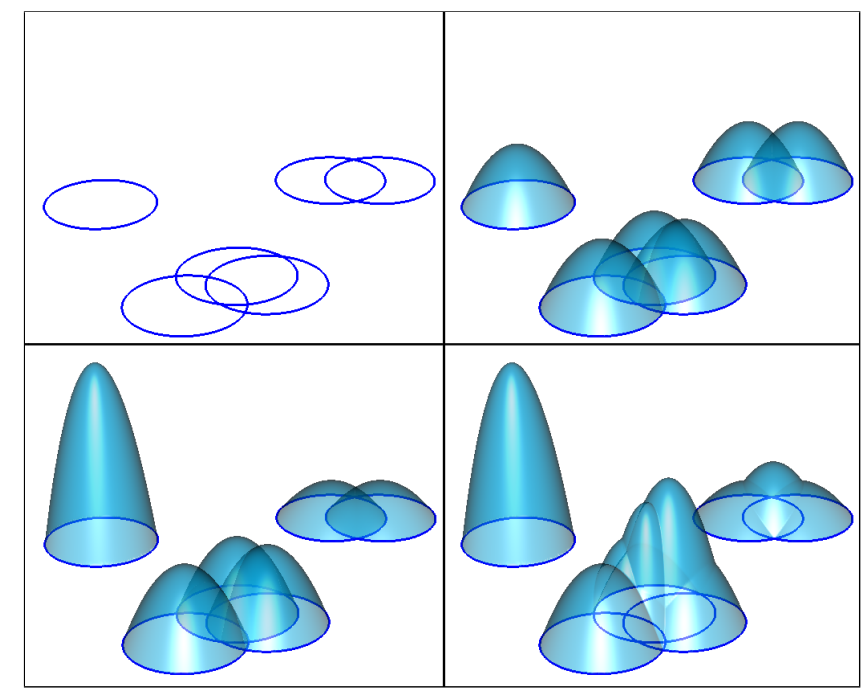

Figure 2. Illustration of the modeling approach for precipitation amounts: (1) generating circular precipitation cells (top left), (2) assigning a response function to each cell (top right), (3) scaling the response functions (bottom left), (4) summing scaled response functions to obtain precipitation amounts (bottom right).

Sect. 3.1, the process of cell centers $\left\{X_{i}, i=1, \ldots, Z\right\}$ is conditionally a Poisson process the distribution of which is completely determined by the local intensities $a_{1}, \ldots, a_{503}$. These intensities and the cell radius, $r$, were already computed from available point probabilities, see Kriesche et al. (2015). Next, we turn our attention to the scaling variables. Let $\left\{e_{t}, t \in W\right\}$ and $\left\{v_{t}, t \in W\right\}$ denote the deterministic fields of expectations and variances of precipitation amounts, i.e., $e_{t}=\mathbb{E} \Gamma_{t}$ and $v_{t}=\operatorname{var} \Gamma_{t}$ for all $t \in W$. Recall that in Sect. 2 we explained how the expectations $e_{s_{1}}, \ldots, e_{s_{503}}$ and variances $v_{s_{1}}, \ldots, v_{s_{503}}$ of the precipitation amounts at the locations $s_{1}, \ldots, s_{503}$ can be determined from available data. Intuitively, the distributions of $C_{1}, \ldots, C_{503}$ should be chosen in such a way that the expectations and variances of $\Gamma_{s_{1}} \ldots, \Gamma_{s_{503}}$ coincide with the available data $e_{s_{1}}, \ldots, e_{s_{503}}$ and $v_{s_{1}}, \ldots, v_{s_{503}}$. First, we show how these data are used to compute expectations and variances of $C_{1}, \ldots, C_{503}$. Let $c_{i}=\mathbb{E} C_{i}$ for $i=1, \ldots, 503$. By using Campbell's formula for point processes (Chiu et al., 2013) and some elementary calculations we get

$$
e_{t}=\sum_{i=1}^{503} c_{i} a_{i} \int_{V\left(s_{i}\right) \cap b(t, r)}\left(1-\frac{|x-t|^{2}}{r^{2}}\right)^{p} d x \quad \text { for } t \in W
$$

By putting $t=s_{1}, \ldots, s_{503}$ we get a system of 503 linear equations with the unknowns $c_{1}, \ldots, c_{503}$ for any fixed value of $p$. This system of equations is solved under the constraint $c_{1}, \ldots, c_{503} \geq 0$ using the values of $e_{s_{1}}, \ldots, e_{s_{503}}$. In a similar way the variances $\tilde{c}_{i}=\operatorname{var} C_{i}$ for $i=1, \ldots, 503$ are fitted. By doing some simple calculations and using a covariance formula for Poisson processes, see Beneš and Rataj (2004) for a stationary version, we obtain the following expression for the variance $v_{t}$ of $\Gamma_{t}$ :

$$
v_{t}=\sum_{i=1}^{503} \tilde{c}_{i}\left[a_{i} \tilde{I}\left(s_{i}, t\right)+a_{i}^{2} I^{2}\left(s_{i}, t\right)\right]+\sum_{i=1}^{503} c_{i}^{2} a_{i} \tilde{I}\left(s_{i}, t\right) \quad \text { for } t \in W,
$$

where

$$
I\left(s_{i}, t\right)=\int_{V\left(s_{i}\right) \cap b(t, r)}\left(1-\frac{|x-t|^{2}}{r^{2}}\right)^{p} d x \quad \text { and } \quad \tilde{I}\left(s_{i}, t\right)=\int_{V\left(s_{i}\right) \cap b(t, r)}\left(1-\frac{|x-t|^{2}}{r^{2}}\right)^{2 p} d x .
$$

Again, we put $t=s_{1}, \ldots, s_{503}$ to get a linear system of equations (based on (7)) with the unknowns $\tilde{c}_{1}, \ldots, \tilde{c}_{503}$ for any fixed value of $p$. As before, this is solved under the constraint $\tilde{c}_{1}, \ldots, \tilde{c}_{503} \geq 0$. Now that the expectation and variance of each scaling variable $C_{i}$ are known, a parametric distribution can be fitted to $C_{i}$, where we suggest to use one of the following: beta prime, gamma, inverse gamma, inverse normal and log-normal. These distributions seem to be the most suitable since they are defined on the nonnegative real line and their parameters can be represented as closed functions of expectation and variance, which is crucial for model fitting. A recommendation for the choice of the shape parameter $p$ and the type of distribution for the scaling variables is given in Sect. 4 below. 

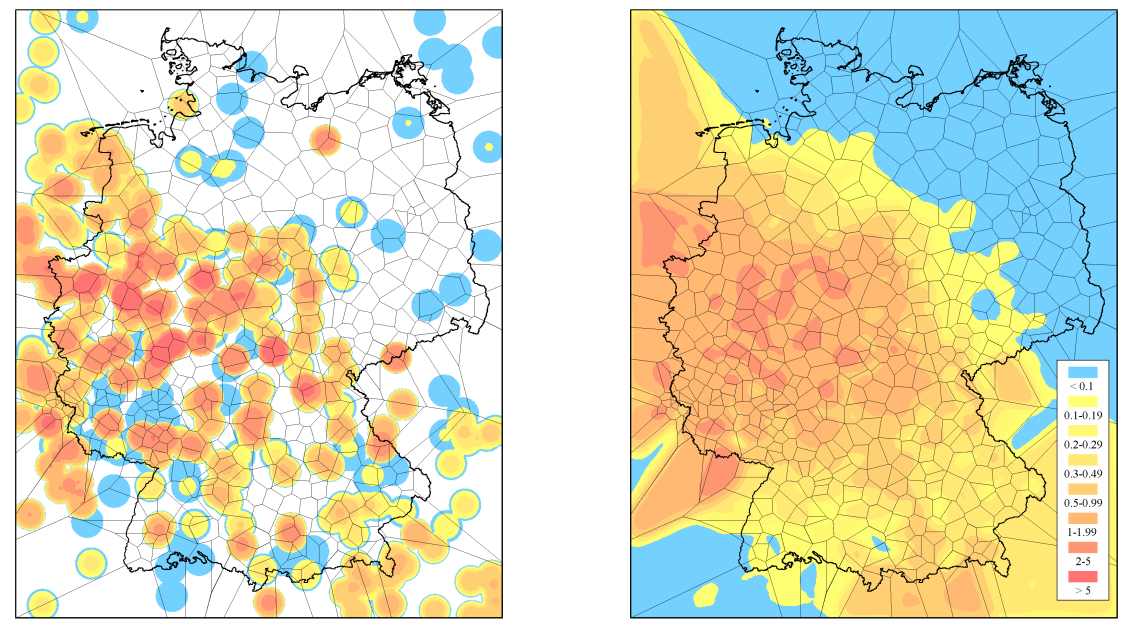

Figure 3. Simulation results for June 13, 2012, 05-06 UTC: typical realization of the fitted shot-noise model of precipitation amounts given in (5) (left) and estimated mean field of simulated precipitation amounts based on 5000 realizations (right).

\subsection{Computation of area probabilities for precipitation exceeding a certain threshold}

According to the combined model for precipitation cells and amounts a computation of area probabilities for precipitation exceeding a certain level is now possible. For $u \geq 0$ and a test area $B \subset W$ the probability for the occurrence of precipitation of more than $u \mathrm{~mm}$ somewhere within $B$ is represented as $P\left(\max \left\{\Gamma_{t}, t \in\right.\right.$ $B\} \geq u$ ). In particular, a corresponding point probability for a single location $t \in W$ can be obtained by putting $B=\{t\}$. Unfortunately, no closed formula for the representation of such point or area probabilities is known. However, an estimation of these probabilities by repeated simulation of the proposed precipitation model is possible. In order to do so, a large number of realizations of the union set $M$ of precipitation cells and of the scaling variables $C_{1}, \ldots, C_{503}$ have to be generated (we recommend 5,000 runs, which can be done in a reasonable computation time), and the probabilities to be determined are then estimated as relative frequencies of the considered events among the 5,000 realizations. A typical realization for one forecast step together with the mean field of simulated precipitation amounts is illustrated in Fig. 3. Both illustrations correspond well with the underlying data shown in Fig. 1.

\section{CHOICE OF RESPONSE FUNCTIONS AND DISTRIBUTION OF SCALING VARIABLES}

We give a recommendation how to choose the shape parameter $p$ of the response function and the distribution type of the scaling variables $C_{1}, \ldots, C_{503}$ based on a comparison of available data and point probabilities computed according to our model. In the following, we consider $p=0.5$ (upper half of unit ball), $p=1$ (Epanechnikov kernel), $p=2$ (biweight kernel), $p=3$ (triweight kernel) and $p=4$ as potential values of the shape parameter $p$ as well as the following parametric distributions for scaling variables: beta prime, gamma, inverse gamma, inverse normal and log-normal. For each value of $p$, each distribution and each threshold $u \in\{0.1,0.2,0.3,0.5,0.7,1,2,3,5,10,15\}$, point probabilities for all weather stations inside the boundaries of Germany and all forecast steps are estimated. Three scores are computed to compare these estimated probabilities with the available data: bias, correlation coefficient and mean squared difference (msd). Due to the lack of space only some selected scores will be presented here. In all considered cases correlation and msd show the best performance for the gamma distribution, although the effect of changing the type of distribution appears to be minor. A more noticeable effect is observed when varying the value of the shape parameter $p$ of the response function. In Table 1 the msd (summed over all weather stations) is given for selected thresholds and all considered shape parameters, where always the gamma distribution is used to model the scaling variables. Choosing $p=0.5$ is obviously not suitable since almost all computed msds are significantly higher than for other shape parameters. We suggest to use the scaled Epanechnikov kernel $(p=1)$ as a response function for precipitation amounts since msd's (and also correlation coefficients) show the best performance for almost all thresholds. For the highest thresholds $(u=5,10,15)$ the scaled biweight kernel seems to be a reasonable choice as well. The bias does not show any significant difference for the tested model configurations. However, the impact of changing the distribution type of the scaling variables as well as the shape parameter (except of $p=0.5$ ) seems to be low. Therefore, also a validation of our model using 
B. Kriesche et al., A model-based approach to the computation of area probabilities ...

Table 1. Mean squared differences of point probabilities obtained from data and estimated using our model with the gamma distribution modeling the scaling variables for different shape parameters and thresholds.

\begin{tabular}{c|cccccc} 
threshold in mm & 0.1 & 0.3 & 0.7 & 2 & 5 & 15 \\
\hline $\operatorname{msd}$ for $p=0.5$ & $2.6 \cdot 10^{-2}$ & $2.5 \cdot 10^{-2}$ & $1.3 \cdot 10^{-2}$ & $2.1 \cdot 10^{-3}$ & $1.2 \cdot 10^{-4}$ & $8 \cdot 10^{-7}$ \\
\hline $\operatorname{msd}$ for $p=1$ & $3.3 \cdot 10^{-3}$ & $2.1 \cdot 10^{-3}$ & $1.16 \cdot 10^{-3}$ & $2.7 \cdot 10^{-4}$ & $3.2 \cdot 10^{-5}$ & $2.27 \cdot 10^{-6}$ \\
\hline $\operatorname{msd}$ for $p=2$ & $3.5 \cdot 10^{-3}$ & $2.4 \cdot 10^{-3}$ & $1.3 \cdot 10^{-3}$ & $2.71 \cdot 10^{-4}$ & $3.13 \cdot 10^{-5}$ & $2.25 \cdot 10^{-6}$ \\
\hline $\operatorname{msd}$ for $p=3$ & $4.2 \cdot 10^{-3}$ & $2.9 \cdot 10^{-3}$ & $1.48 \cdot 10^{-3}$ & $2.81 \cdot 10^{-4}$ & $3.4 \cdot 10^{-5}$ & $2.32 \cdot 10^{-6}$ \\
\hline $\operatorname{msd}$ for $p=4$ & $5.1 \cdot 10^{-3}$ & $3.4 \cdot 10^{-3}$ & $1.7 \cdot 10^{-3}$ & $3 \cdot 10^{-4}$ & $4 \cdot 10^{-5}$ & $2.4 \cdot 10^{-6}$
\end{tabular}

radar data, which will be performed in a forthcoming paper, should be taken into consideration when choosing the model configurations.

\section{CONCLUSION}

We proposed a method for the computation of area probabilities for the occurrence of precipitation amounts exceeding a given threshold. Our approach is based on a combined model for precipitation cells and precipitation amounts and all model parameters can be computed algorithmically based on point probabilities for an ensemble of weather stations. Furthermore, non-stationarity effects are taken into account. In contrast to previous approaches considered in the literature, our method allows for an automated computation of weather warnings. A detailed validation of estimated area probabilities using radar data will be performed in a forthcoming paper. Further research will also include refinements of the precipitation model such as elliptical precipitation cells, clustering of precipitation cells and different cell types.

\section{ACKNOWLEDGEMENT}

The authors gratefully acknowledge the financial supports from the German Academic Exchange Service (DAAD) and the Czech Ministery of Education, project 7AMB14DE006.

\section{REFERENCES}

Beneš, V. and J. Rataj (2004). Stochastic Geometry: Selected Topics. Boston: Kluwer Academic Publishers.

Chiu, S. N., D. Stoyan, W. S. Kendall, and J. Mecke (2013). Stochastic Geometry and its Applications (3rd ed.). Chichester: J. Wiley \& Sons.

Kriesche, B., R. Hess, B. K. Reichert, and V. Schmidt (2015). A probabilistic approach to the prediction of area weather events, applied to precipitation. Spat. Stat. 12, 15-30.

Krzysztofowicz, R. (1998). Point-to-area rescaling of probabilistic quantitative precipitation forecasts. J. Appl. Meteorol. 38, 786-796.

Majewski, D., D. Liermann, P. Prohl, B. Ritter, M. Buchhold, T. Hanisch, G. Paul, W. Wergen, and J. Baumgardner (2002). The global icosahedral-hexagonal grid point model GME: description and high-resolution tests. Mon. Weather Rev. 130(2), 319-338.

Onof, C. J., R. E. Chandler, A. Kakou, P. J. Northrop, H. S. Wheater, and V. S. Isham (2000). Rainfall modelling using poisson-cluster processes: a review of developments. Stoch. Environ. Res. Risk Assess. 14(6), 384-411.

Rodriguez-Iturbe, I., D. R. Cox, and P. S. Eagleson (1986). Spatial modelling of total storm rainfall. Proc. Royal Soc. Lond. A 403(1824), 27-50.

Segond, M.-L. and C. J. Onof (2009). Modelling of space-time rainfall for three UK regions. Proc. ICE Water Manag. 162(2), 147-158.

Wheater, H. S., R. E. Chandler, C. J. Onof, V. S. Isham, E. Bellone, C. Yang, D. Lekkas, G. Lourmas, and M.-L. Segond (2005). Spatial-temporal rainfall modelling for flood risk estimation. Stoch. Environ. Res. Risk Assess. 19(6), 403-416.

Wilks, D. S. (1995). Statistical Methods in the Atmospheric Sciences. San Diego: Academic Press. 